\title{
Experiences of women with the support they received from their birth companions during labour and delivery in Malawi
}

\author{
Esther Kungwimba ${ }^{1}$, Address Malata $^{2}$, Alfred Maluwa $^{3^{*}}$, Ellen Chirwa $^{4}$ \\ ${ }^{1}$ Malawi College of Health Sciences, Blantyre, Malawi \\ ${ }^{2}$ Department of Maternal and Child Health, University of Malawi, Kamuzu College of Nursing, Lilongwe, Malawi \\ ${ }^{3}$ Research Directorate, University of Malawi, Kamuzu College of Nursing, Lilongwe, Malawi; \\ *Corresponding Author: aomaluwa@kcn.unima.mw \\ ${ }^{4}$ Department of Maternal and Child Health, University of Malawi, Kamuzu College of Nursing, Blantyre, Malawi
}

Received 28 October 2012; revised 29 November 2012; accepted 6 December 2012

\section{ABSTRACT}

A study was conducted to describe the experiences of primiparous women with the support they received from their birth companions during labour and delivery in Malawi. The study design was descriptive and utilized qualitative data collection and analysis method on a sample of 20 primiparous women. The women were recruited from the postnatal ward of Bwaila hospital and were interviewed regarding their experiences on the support they received from their birth companions during labour and delivery using an open ended interview guide. Data was manually analyzed using content analysis. Primiparous women described the support they received from their birth companions as useful and beneficial. Birth companions provided advice, physical, emotional and spiritual support to the women during their labour and delivery. In addition, the birth companions were viewed as guardians of the women during labour and delivery. Results however, show that some women were not properly assisted by their companions because both the mothers and companions lacked knowledge on birth companionship. The results further show that birth companions play important roles during the birth and delivery of primiparous women and thereby improving birth outcomes. Therefore, there is a need to train the companions regarding support to a woman during labour and delivery. Primiparous women also need to be trained during antenatal care education so that they properly understand the roles of a birth companion as opposed to those of the midwives.
Keywords: Primiparous Woman; Birth Companion; Labour; Delivery Support; Antenatal Clinic Education; Birth Experience

\section{INTRODUCTION}

Labour and delivery experience is one of the most significant events in a woman's life [1] because first time experience with labour and delivery brings anxiety to women [2]. Primigravid women have little or no understanding of labour and delivery process hence they need concrete support, companionship and empathy. Support from family and friends may help to meet the needs of the labouring first time mother that the midwife may not be able to address, thus facilitating positive birth experience for the primiparous woman [3]. The presence of birth companions during childbirth ensures that a woman is not left alone during this intensely stressful and frightening time in her life [4]. Birth companions are non medical people who provide information, physical and emotional support to women during labour and delivery [5].

Support to women during childbirth from a birth companion is practiced worldwide, including United States of America, Canada, United Kingdom, some parts of western and northern Europe [6,7], Mexico, parts of Latin America, Asia and Africa [8,9]. Studies have shown that various individuals serve as birth companions and these include: doulas and lay people like mothers, sisters, male partners, friends, and siblings [10]. A doula is a woman who is trained and experienced in childbirth and provides continuous information in addition to physical and emotional support to a woman during labour, birth, and the immediate postpartum period [11]. Support from doulas is mainly practiced in developed countries including the United States of America, United Kingdom, Australia, some parts of the western and northern Europe 
and South Africa [10].

In many developing countries especially in Africa, a birth companion is usually a relative because many people cannot afford to pay doulas. In Malawi, a study conducted by Banda, et al., [9], found that relatives such as mothers, mothers in law, sisters, grandmothers and friends are utilised as birth companions. However, unlike other countries where women in labour choose in advance their companion, birth companions in Malawi are not chosen by the women themselves and are hence not prepared for this role in advance. The common practice is that any woman who escorts a woman in labour to a hospital becomes a birth companion. There is therefore no preparation or orientation of the birth companions on their roles.

Shortage of staff is prevalent in all hospitals in Malawi consequently the labouring women have little contact with the midwives [12] as the health workers have to attend to many women in labour at the same time. Consequently, there is a need for relatives to support the women during labour. Companionship during labour and delivery was found to be beneficial mainly for psychological and physical support and for providing assistance to health care workers [13] hence, the practice is advocated in the reproductive health standards of the country [12]. It was therefore recommended that birth companionship be introduced in hospitals of the country and currently the practice is being advocated for up scaling in all the hospitals of the country. In line with the reproductive health standards, Bwaila hospital introduced birth companionship in 2009. However, little is known about the experience and perception of primiparous women with birth companionship. The aim of this study was therefore to describe the experience of primiparous women towards the support they received from their birth companions during their labour and delivery.

\section{METHODS}

\subsection{Design}

The study employed a descriptive design that utilized qualitative data collection and analysis method.

\subsection{Setting}

This study was conducted at Bwaila hospital in the central region of Malawi. The hospital is one of the largest government referral health facilities for both outpatient and inpatient pregnant women.

\subsection{Sample}

Data saturation was reached after interviewing 18 primiparous women but 2 more were added to validate the results, hence the final sample size was 20. Primiparous women in the postpartum ward were randomly sampled and only those that met the inclusion criteria were invited to participate in the study $[14,15]$.

\subsection{Inclusion and Exclusion Criteria}

The study included all women who; were aged between 15 and 30 years, delivered for the first time, received continuous support from a birth companion (male or female) during labour and delivery, delivered vaginally a live baby, were physically able to participate, spoke the vernacular language or English, and did not have a sick or pre-term baby. The study excluded all women who had delivered more than once, had not received support during labour and delivery or delivered through a caesarean section, were sick or had a sick child.

\subsection{Data Collection}

Data was collected from August 2011 to September 2011 in a postnatal ward of Bwaila hospital with assistance of the midwives on duty. In depth interviews were conducted using an open ended interview guide. Interviews were recorded using a digital recorder. Areas of focus during data collection were on the knowledge of participants regarding birth companionship, type of support the participants received from their birth companions and their perception of the support.

\subsection{Data Analysis}

Tape recorded data was transcribed verbatim in the vernacular language and then translated into English. Back translation was done by an independent person to validate the content of the message and to ensure retention of the original information. Data was manually analyzed using content analysis. This included review of data to gain a feeling of the study topic's inherent meanings. Significant statements were extracted, meanings were formulated, similar meanings were isolated and grouped into a cluster of themes, exhaustive descriptions of the experiences of the primiparous women were written down and identification of fundamental structures for each exhaustive description was done. The final stage required a return to each woman to validate the interpretations of the findings (member check). However, this was not possible because by the time the analysis was completed the women had already been discharged from the hospital. Instead, the study results were given to two independent persons who independently analysed the data and verified the themes with those in the study to ensure trustworthiness of the results.

\subsection{Ethical Considerations}

The study was approved by College of Medicine Re- 
search and Ethics Committee (COMREC). Informed consent was obtained from each participant and participation was voluntary with an option of withdrawing at any stage of the study if the participants wanted to withdraw. All the information was treated confidentially and participants were identified by code numbers rather than their names to ensure confidentiality.

\section{RESULTS}

A total of 20 primiparous women participated in the study. The demographic characteristics of the women are summarized in Table 1. Half of the women $(50 \%, \mathrm{n}=10)$ were aged between 20 and 25 years and most of them (85\%, $n=17$ ) were married. Regarding their educational status, $45 \%, \mathrm{n}=9$ had some secondary school education and $30 \%, \mathrm{n}=6$ had completed their secondary school education. Over half of the women $(55 \%, n=11)$ were house wives (Table 1).

Two themes, knowledge of birth companionship and support from birth companions emerged from the participants' narrations.

\subsection{Knowledge of Participants on Birth Companions}

The narrations of the participants regarding their knowledge of birth companionship led to the emergence of two subthemes; source of knowledge and perceived roles of the birth companions.

\subsection{Source of Knowledge}

Most participants (14 out of 20) knew that they were supposed to bring a birth companion to be with them during their labour and delivery. For those who knew, (9 out of 14) learnt this from their friends, relatives, and

Table 1. Demographic characteristics of the women who participated in the study.

\begin{tabular}{cccc}
\hline Characteristic & Category & Number & Percentage \\
\hline \multirow{3}{*}{ Age } & $15-19$ & 8 & 40 \\
& $20-25$ & 10 & 50 \\
& $26-30$ & 2 & 10 \\
Marital status & Single & 2 & 10 \\
& Married & 17 & 85 \\
& Divorced & 1 & 5 \\
Education & Some primary & 2 & 10 \\
& Completed primary & 2 & 10 \\
& Some secondary & 9 & 45 \\
& Completed secondary & 6 & 30 \\
& Tertiary & 1 & 5 \\
& Business & 5 & 25 \\
& House wife & 11 & 55 \\
& Student & 2 & 10 \\
& other & 2 & 10 \\
\hline
\end{tabular}

marriage councillors. Another important source of information for 5 out of 14 participants was the antenatal clinic. Woman \#8 explained:

The marriage councillors advised me that when labour starts I should have somebody to escort me to the hospital. It was also mentioned at the antenatal clinic.

Despite the knowledge regarding companionship when going to the hospital, some participants (3 out of 14) did not know that they would be with their companions in the labouring room as shared by woman \#4:

Councillors came to advise me about pregnancy and they also told me that when coming to the hospital I should bring a companion. But I did not know that she will be with me during labour and delivery.

\subsection{Perceived Roles of the Birth Companions}

All the sources of information gave similar information on the roles of birth companions. According to 11 out of 14 women the roles of birth companions included caring for the woman during labour and delivery especially when the midwife was not there, to receive the baby after delivery, and to observe every activity during labour and delivery.

They said you should have a guardian because there are some issues that she should observe during delivery of the baby. She should know the progress, she should have enough evidence on how you are performing during delivery, she should observe and have first hand information on everything that happens. Woman \#3.

\subsection{Support Received from Birth Companions}

The women narrated their experiences from the support they received from their birth companions. The results are reported under two sub themes that emerged, which were; elements of support and desired support.

\subsection{Elements of Support}

The women explained that they received advice, physical, emotional and spiritual support from their birth companions.

\subsection{Advice}

All the women (20) reported to have received sound advice from their companions which was very helpful. Some of the information included how labour progresses, what to do during contractions and signs of the second stage of labour. Woman \#13 narrated as follows:

My mother was advising me on what to do. She was advising me to lie on my sides, to turn, so I was listening to my mother. I was advised against screaming because 
it leads to exhaustion, I was advised by my mother and not the nurses. She said sometimes you feel very hot or cold. Sometimes you may be in pain so she advised me that when in pain I should hold breath so that the breath should go down and push the baby down. When the pain is over I should rest. My mother kept asking me, how I was feeling, so I was explaining to her how I felt and she was advising me what to do. Similarly when I had experienced something strange I asked her and she told me what to do. But there were no nurses.

The other important aspect of birth companionship was that the primiparous women were advised about appropriate behaviour during labour. Some of the women (7 out of 20) explained that they were advised against being troublesome but to listen to what they were being advised by the doctors and their birth companions.

She told me what to do when I reached a certain stage. She advised me to do my best for the baby to come out. She said... laughing..." my daughter this is your first pregnancy, all of us have had our babies through the same process so don't think there will be something strange. My mother delivered 10 children through the same process.” Participant \#2.

For half of the women (10 out of 20) their birth companions were a source of good advice and encouragement as narrated by primiparous woman \#17.

When I complained that I was feeling pain she told me that she also went through the same process, and that I just needed to be strong if I wanted to go home with a baby, I did not need to cry though in pain. So I followed the advice and kept quiet.

\subsection{Physical Support}

Birth companions were a source of physical support to the primiparous women. The physical support included; massaging the back, provision of clean clothes, soap and warm water to bath, washing clothes and beddings, and wrapping the baby after delivery. Birth companions also helped some women to get into comfortable positions during the first stage of labour and in the right position during second stage of labour as shared by primiparous woman \#6: During delivery, she was holding my head to raise me a little bit and assisted me to push effectively.

Some birth companions (for 6 out of 20 women) ensured that the primiparous women they were assisting had enough fluids to drink and food to eat especially porridge. They also assisted with wiping the beds when they got wet and escorted them to the toilet.

When I wanted to go to the toilet she held me by hand to and from the toilet, she made my bed so that I could sleep there comfortably. When the nurse said that I needed food because I was hungry, my mother prepared porridge for me to eat-primiparous women \#20.

\subsection{Emotional Support}

All the primiparous women (20) explained that their companions were useful because they provided emotional support. The support included chatting and praising them for good performance especially during the second stage of labour. The presence of the birth companions provided company and relief from anxiety to the women who had no experience of labour.

I was very happy with my companion because at first I was afraid, I did not know what to do. You know people talk a lot. Someone told me that usually women are left alone without any nurse and sometimes they give birth while alone. I was worried. But when I knew that I would be with my mother in-law I was very happy because I knew I would not be alone, I would be asking her questions, I was also happy that she would be observing the progress, and also that I had somebody to chat with when the nurses were not around-primiparous women \# 5 .

\subsection{Spiritual Support}

Some primiparous women (2 out of 20) were encouraged to pray for themselves. In addition, their companions prayed with them for safe delivery as shared by woman \# 11. She was also encouraging me spiritually. I am a catholic by faith so we were praying.

\subsection{Desired Support}

Despite the appreciation of the support that the women received from their birth companions, some (13 out of 20) women explained that they did not receive the desired support from their companions. Some women's birth companions (2 out of 13) refused to escort the labouring women to the toilet or to be close to them so they could hold on to their companions. The companions for some women (3 out of 13) refused to assist the labouring women with turning, conducting back massaging and even providing food as narrated by primiparous woman \# 8:

Oh yes, but I wanted her to be near me so I could hold her during contractions, assist me with turning because it was very difficult for me to turn but she refused.

Primiparous woman \# 4 had a negative experience with her companion who even refused her to take food:

I wanted food but my companion did not give me, she said I will eat after delivery, but I really wanted to eat food or drink tea because I was hungry. So I said mmmm let me take the advice since she went through this process already, she is the one who knows better than I do.

Some women (3 out of 20) reported extremely negative experiences with their companions. The birth companions for these women shouted at them while in labour 
instead of rendering the desired support. Primiparous woman \# 17 stated that her birth companion even threatened to abandon her in the labour ward:

I was in pain and I asked her to allow me to go to the toilet to urinate. Surprisingly her response was that she was going home and will leave me alone. She left but came back after a nurse pleaded with her. She told me that I was troublesome. You know it was my first time... smiling... On the second time, during a contraction I wanted to wake up but she advised me to lie down. I told her that I was failing to lie down, and she said again that she was going to abandon me.

For those with negative experiences, most of them (7 out of 13) explained that their companions discouraged them from drinking water while in labour. There is a traditional belief that drinking water while in labour stops the contractions. For some women, (2 out of 13) wished their companions had poured cold water on them or had wiped their bodies with a wet cloth if they could not drink water because they were feeling very hot, but their companions refused to assist them.

Yes I wanted to drink water but I did not take the water because the councillors advised me that when I take water during labour the contractions would be ineffective. My companion was also afraid to give me water because of the same reason, but mmmm I was not okay I was very thirsty I wanted just a small amount of water to moisten my throat which was so dry, but mmmm I did not take water until delivery of the baby-primiparous woman \# 10.

\section{DISCUSSION}

The primiparous women had some education and the majority of them had attained secondary education. Although education has an empowering effect on women through the broadening of their horizons and making them aware of available opportunities [16], results show that some primiparous women in this study did not get the care they wanted because their expectations were wrong due to lack of proper information regarding companionship during labour. The primiparous women did not know the type of support they were supposed to receive from their birth companions and on the other hand, the companions did not know the type of support they were expected to provide to the primiparous women.

\subsection{Knowledge on Birth Companions}

Women in this study used external sources of information on birth companionship such as relatives, friends, and councillors despite the fact that companionship during labour is a component of routine antenatal care in Malawi. Midwives at the antenatal clinic did not provide adequate information to the women. These results have an implication on the expectations of the women regarding their care. In contrast, results from other countries, for example in Hong Kong [17] and Sweden [18], the main sources of childbirth information are the midwives during antenatal care education. Therefore in Malawi, there is information gap regarding birth companionship which needs to be filled up during antenatal care clinics.

Some of the narrations from the women suggest that they perceived birth companions to play the roles of nurses and midwives during their absence. Birth companions do not take the role of a midwife [19] but focuses on the mother's comfort and wishes during childbirth [11]. Birth companion supports a labouring woman by providing physical comfort and by staying with her continuously throughout labour and delivery [20]. Furthermore, birth companions should not be viewed as guards because support to women during labour becomes ineffective if they assume that role.

\subsection{Support Received from the Birth Companions}

All the participants in this study depended on their birth companions for advice about labour and delivery as also reported in Sweden [21]. The results show that women during labour and delivery are receptive to the suggestions given to them. Most of the women could not insist on something they wanted during labour and delivery but took the advice of their birth companions. Some of the advice prevented them from expressing their feelings and concerns. In some cases, the advice was threatening and provoked anxiety. This is contrary to the aim of support during labour and delivery in which women in labour need to feel respected, loved, nurtured, encouraged and reassured.

Results show that some of the advice given to the women by their companions, for example, withholding breath during contractions to push the baby down during the first stage of labour contradicted the advice given by midwives. The midwives advised women to breathe through the mouth during contraction when in the first stage of labour. The primiparous women were therefore confused on which advice to take. In most cases they preferred advice from the companion who was always at the bedside. However such advice could lead to oedema of the cervix, prolonged labour and trauma to the cervix during delivery. Therefore, there is a need for proper training of birth companions so that they give correct information to the women.

Due to the labour pains, the women could not perform some self care activities hence relied on their birth companions for support. Results in this study show that some of the birth companions provided some of the physical support like back massage, provision of food and fluids, 
washing clothes, assisting with walking around and escorting the women to the toilet. These results are similar to the findings of studies from Sweden $[21,22]$.

The results show that the presence of birth companions during labour and delivery was perceived to be very important to the primiparous women. The women were anxious and afraid of labour pains, bad attitudes of midwives, and being left to deliver their babies alone. To be anxious and afraid of labour was normal because they had not previously experienced childbirth. In addition, they had received threatening information from their friends, relatives and councillors about labour and delivery. When a woman faces childbirth for the first time, she often feels anxious, because coping with labour pain is widely viewed as an anxious moment [2]. The presence of birth companion made all the participants feel safe, including those who had bad relationships and had developed bad attitudes towards their birth companions. Similarly, Jamas et al. [23] reported that emotional support that women got from their birth companions resulted in feelings of security. Another study also identified emotional security as one of the reasons women wanted a birth companion during childbirth [13].

In this study, results show that the primiparous women needed birth companions who could be there just for them and to render the physical, emotional and spiritual care. In addition the participants expected their companions to praise them for good performance and also to reassure and encourage them as also reported by other studies [19]; massaging their backs, helping them into comfortable positions and with relaxation techniques [24]; making sure that they have adequate food and fluids, and reminding them to go to the toilet regularly [25]. Women in labour should be provided with the required information and services, and be accepted as they are. Furthermore, birth companions should be non judgemental and should ensure that the primiparous women feel welcome and comfortable [26]. This kind of support helps to build trust, strong relationship and a feeling of safety hence reducing anxiety and level of pain the mother experiences during childbirth.

Knowledge gap was identified when some women narrated that their companions refused them to drink and eat while in labour. Labour requires an enormous amount of energy. As the length of labour and delivery cannot be predicted, the source of body energy needs to be replenished to ensure foetal and maternal well-being [27]. Restriction of oral intake during labour and delivery is very stressful and can lead to dehydration and ketosis. Oral intake may be a form of comfort that a woman may require to labour successfully [28]. Traditional beliefs especially on the restriction of drinking cold water during labour also played a part on the provision of inadequate support. The American society of Anaesthesiologists [29] recommends oral intake of clear liquids for women with uncomplicated labour and these include water, tea, coffee, and fruit juices. There is no evidence that cold water can cause ineffective uterine contractions, it is just a belief that causes unnecessary stress to women during labour and delivery.

In general, the companions rendered good support. Deficiencies in the support that was rendered could be attributed to lack of knowledge on the roles of birth companions by both the primiparous women and their birth companions. Participants could not explain properly the roles of their birth companions. Some companions were not ready to take up their role because they were not prepared both physically and psychologically in advance, hence tended to be emotional on petty issues. Banda et al. [13] reported that unwilling companions are less likely to play the role expected from a labour companion and the supported woman may not benefit from the companionship. Furthermore, there was no support for the birth companions from the midwives. Support to the birth companions by midwives could have improved the support the birth companions rendered to the participants. Thus there is a need for the midwives to avail themselves to the birth companions.

\section{CONCLUSION}

Support that was rendered to the primiparous women from their birth companions was beneficial. The birth companions provided physical, emotional and spiritual support to the primiparous women that assisted them to go through the labour process successfully. However, some of the advice that was given to the women in labour was not correct and some birth companions were unable to provide the required support due to lack of knowledge on their roles. The women at times expected too much from their birth companions and did not differentiate some of the roles of a birth companion and those of a midwife. On the other hand, the midwives did not render the needed support to the birth companions, thus some of the women did not perceive the roles of their companions as being helpful but obeyed them to avoid creating an impression that they were troublesome. Therefore, there is a need to provide adequate information to pregnant women about companionship during antenatal care education. In addition, birth companions need to be properly trained on how to assist women in labour and there is a need for the midwives to support the birth companions so that the labouring women are properly assisted by their companions.

\section{LIMITATION OF THE STUDY}

The study was conducted at one site only due to limited time and financial resources. As such the findings 
may not give a general representation of the experiences of all primiparous women who receive support from birth companions during labour and delivery in Malawi.

\section{ACKNOWLEDGEMENTS}

The study was conducted as part of the senior author's Master of Science degree in Midwifery at the University of Malawi, Kamuzu College of Nursing with a scholarship from Global Fund through National AIDS Commission in Malawi. The preparation of the manuscript for publication was funded by the University of Tromso, Norway and the Agency for Norwegian Development Cooperation.

\section{REFERENCES}

[1] Khresheh, R. (2010) Support in the first stage of labour from a female relative: The first step in improving the quality of maternity services. Midwifery, 26, e21-e24. doi:10.1016/j.midw.2008.11.003

[2] Cheung, W., Ip, W.Y. and Chan., D. (2007) Maternal anxiety and feelings of control during labour: A study of Chinese first time pregnant women. Midwifery, 23, 123130. doi:10.1016/j.midw.2006.05.001

[3] Melender, H. (2006) What constitutes a good childbirth? A qualitative study of pregnant Finnish women. Journal of Midwifery and Women's Health, 51, 331-339. doi:10.1016/j.jmwh.2006.02.009

[4] Bakhta, Y. and Lee, R.H. (2010) A survey of Russian women regarding the presence of a companion during labour. International Journal of Gynaecology and $\mathrm{Ob}$ stetrics, 109, 201-203. doi:10.1016/j.ijgo.2010.01.021

[5] Bruggemann, O.M., Parpinelli, M.A., Osis, M.J.D., Cecatti, J.G. and Neto, A.S.C. (2007) Support to woman by a companion of her choice during childbirth: A randomized controlled trial. Reproductive Health, 4, 5. doi:10.1186/1742-4755-4-5

[6] Brailey, S. (2008) A Swiss birthing centre. Practice Midwife, 11, 27-28.

[7] Campbell, D., Scott, K.D., Klaus, M.H. and Falk, M. (2007) Female relatives or friends trained as labour doulas: Outcomes at 6 to 8 weeks postpartum. Birth, 34, 220227. doi:10.1111/j.1523-536X.2007.00174.X

[8] Murray, M.L. and Huelsman, C.M. (2009) Labour and delivery nursing: A guide to evidence based practice. New York: Springer.

[9] Banda, G.N. (2008) Female support companionship during labour: Effects on infant Apgar score and maternal pain experience. Master's thesis, University of Malawi, College of Medicine, Blantyre. www.medcol.mw/commhealth/mph/dissertations/Thesis

[10] Stein, M.T. (2004) Benefits of a doula at the birth of a child. Paediatrics, 114, 1488-1491.

[11] Papagni, K. and Buckner, E. (2006) Doula support and attitudes of intrapartum nurses: A qualitative study from the patient's perspective. Journal of Perinatal Education, 15, 11-18. doi:10.1624/105812406X92949

[12] Ministry of Health (2004) A Joint Programme of work for a health Sector Wide Approach (SWAp) (2004-2010). Department of Planning, Ministry of Health, Lilongwe.

[13] Banda, G., Kafulafula, G., Nyirenda, E., Taulo, F. and Kalirani, L. (2010) Acceptability and experience of supportive companionship during childbirth in Malawi. BJOJ: An International journal of obstetrics and gynaecology, 117, 937-945.

[14] Polit D.F. and Beck C.T. (2010) Essentials of nursing research: Appraising evidence for nursing practice. 7th Edition, Wolters Kluwer, Lippincott \& Williams \& Wilkins, Philadelphia, 2010.

[15] Burns, N. and Grove, S.K. (2005) The practice of nursing research: Conduct, critique, and utilization. 5th Edition, Elsevier Saunders, St. Louis.

[16] Ratsma and Malongo (2009) Maternal health and human rights. Malawi Medical Journal, 21, 51-53

[17] Chan, L.S.L. and Wong, M.W.L. (2006) The effect of antenatal education program on maternal-foetal outcomes in Hong Kong Chinese women. Hong Kong Journal of Gynaecology, Obstetrics and Midwifery, 6, 10-15.

[18] Bergstrom, M., Kieler, H. and Waldenstrm, U. (2009) Effects of natural childbirth preparation versus standard antenatal education on epidural rates, experience of childbirth and parental stress in mothers and fathers: A randomised controlled multicenter trial. BJOG: An International Journal of Obstetrics and Gynaecology, 116, 11671176.

[19] Newman, B.M. and Newman, P.R. (2009) Development through life: A psychosocial approach. Wadsworth Cangage Learning, New York.

[20] Yuenyong, S. Jirapaet, V. and O’Brien, B.A. (2008) Support from a close female relative in labour: The ideal maternity nursing intervention in Thailand. Journal of Medical Association in Thailand, 91, 253-260.

[21] Lundgren, I. (2010) Swedish women's experiences of doula support during child birth. Midwifery, 26, 173-180. doi:10.1016/j.midw.2008.05.002

[22] Berg, M. and Terstad, A. (2006) Swedish women's experiences of doula support during childbirth. Midwifery, 22, 330-338. doi:10.1016/j.midw.2005.09.006

[23] Jamas, M.T., Hoga, L.A.K. and Tanaka, A.C.A. (2011) Mother's birth experiences in a Brazilian birth centre. Midwifery, 27, 693-699. doi:10.1016/j.midw.2009.10.004

[24] Simkin, P. (2008) The birth partner: A complete guide to childbirth for Dads, Doulas, and all other labour companions. 3rd Edition, Harvard Common Press, New York.

[25] Morhason-Bello, I.O. (2008) Attitude and preferences of Nigerian antenatal women to social support during labour. Journal of Biosocial Science, 40, 553-562. doi:10.1017/S0021932007002520

[26] Atuyambe, L.M. (2008) Adolescent motherhood in Uganda: Dilemmas, health seeking behaviour and coping responses. PhD Thesis, Makerere University, Kampala. http://diss.kib.ki.se/2008/978-91-7409-212-7/thesis.pdf

[27] Chalmers, B., Mangiaterra, V. and Porter, R. (2001) WHO principles of perinatal care: The essential antenatal, perinatal and postpartum course. Birth, 28, 202-207. doi:10.1046/j.1523-536x.2001.00202.x 
[28] Tranmer, J.E., Hodnett, E.D., Hanna, M.E. and Stevens B.J. (2005) The effect of unrestricted oral carbohydrate intake on labour progress. Journal of Obstetrics, Gynaecology and Neonatal Nursing, 34, 319-328. doi:10.1177/0884217505276155
[29] American Society of Anaesthesiologists (2007) Practice guide lines for obstetric anaesthesia: An updated report by the American Society for Anaesthesiologists Task Force on Obstetric Anaesthesia. Anaesthesiology, 106, 843-863. 